\title{
Epidermoid cyst within an intrapancreatic accessory spleen: endosonography and confocal endomicros- copy of an unusual pancreatic cystic lesion
}

A 62-year-old man with a history of a cholecystectomy presented with longstanding right upper quadrant pain. A computed tomography (CT) scan demonstrated a normal spleen with incidental small adjacent splenules and a $2.4 \times 2.3-\mathrm{cm}$ cystic lesion in the tail of the pancreas. The pancreatic cystic lesion (PCL) had not been seen on a CT scan 2 years earlier, while an interim CT scan had revealed a lesion of $1.1 \times 1.1 \mathrm{~cm}$.

An endoscopic ultrasound (EUS) demonstrated a $2.5 \times 2.2-\mathrm{cm}$ anechoic cystic lesion within the pancreatic tail. During EUS, needle-based confocal endomicroscopy (nCLE) demonstrated cellular cords with many red blood cells within the cystic lesion ( $\bullet$ Fig. 1 a; $\bullet$ Video 1 ). Following nCLE, cyst fluid obtained by fine needle aspiration (FNA) revealed non-diagnostic cytology, an amylase of $183 \mathrm{U} / \mathrm{L}$, and a carcinoembryonic antigen (CEA) level of $2663 \mathrm{ng} / \mathrm{mL}$.

The elevated CEA and increasing size of the PCL were key determinants for the patient to undergo a laparoscopic distal pancreatectomy and splenectomy. An ex vivo nCLE examination of the cyst was performed as per the study protocol ( Fig. 1b; $\bullet$ Video 1). Surgical histopathology revealed a benign epidermoid cyst with mural ectopic splenic tissue, compatible with an epidermoid cyst of an accessory spleen ( $\mathbf{F i g . 2}$ ). The diagnosis was confirmed as being an epidermoid cyst in an intrapancreatic accessory spleen (IPAS).

An IPAS can be difficult to distinguish by cross-sectional imaging and is often evaluated for neoplastic potential [1]. This is the first report of in vivo EUS-guided nCLE visualization of an epidermoid cyst within an IPAS. The cyst with its thin epithelium lacked characteristic nCLE features, but the splenic tissue demonstrated cords of cells suggestive of splenic red pulp. Additional features of other common PCLs were not observed [2]. This study adds to the growing body of literature describing EUS-guided nCLE in PCLs.

Endoscopy_UCTN_Code_CCL_1AZ_2AM

Competing interests: None
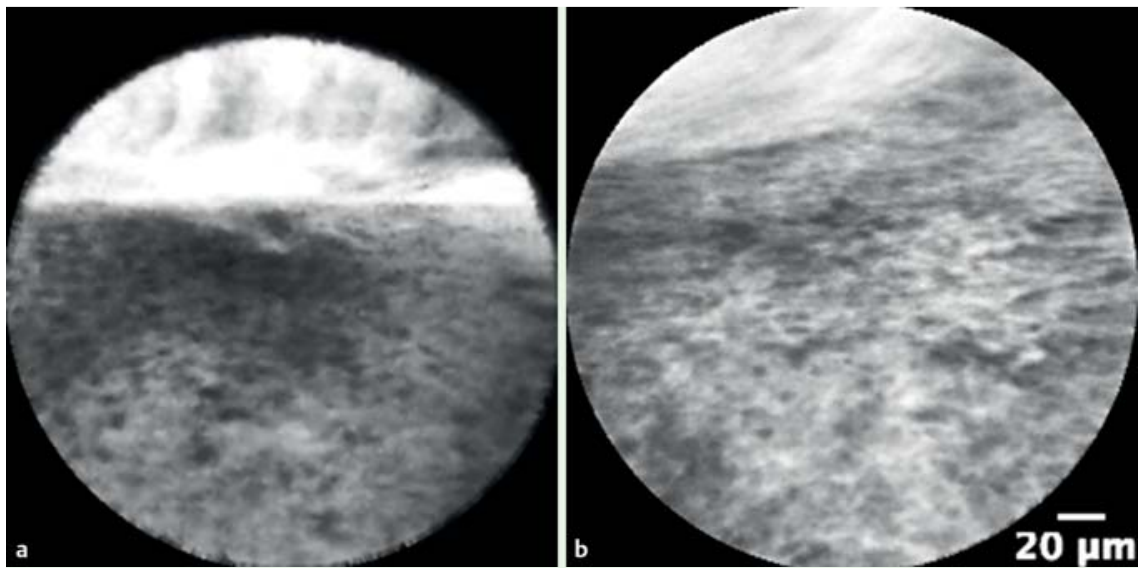

Fig. 1 Appearances of an epidermoid cyst within an intrapancreatic accessory spleen (IPAS) showing cellular cords with multiple red blood cells within the epidermoid cyst: a during endoscopic ultrasound (EUS) in vivo needle-based confocal endomicroscopy (nCLE); b during ex vivo nCLE.

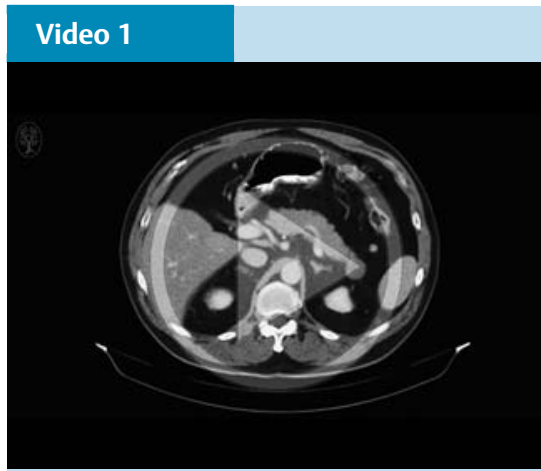

Endoscopic ultrasound (EUS)-guided in vivo confocal laser endomicroscopy (CLE) and postsurgical ex vivo endomicroscopy compared with the final histopathology, which confirmed a benign epidermoid cyst in an intrapancreatic accessory spleen (IPAS).

\section{Rohan M. Modi ${ }^{1}$, Amrit K. Kamboj'} Benjamin Swanson ${ }^{3}$, Darwin L. Conwell $^{4}$, Somashekar G. Krishna ${ }^{4}$

Department of Internal Medicine, The Ohio State University Wexner Medical Center, Columbus, Ohio, USA

2 The Ohio State University College of Medicine, Columbus, Ohio, USA Department of Pathology, The Ohio State University Wexner Medical Center, Columbus, Ohio, USA

${ }^{4}$ Department of Gastroenterology, Hepatology and Nutrition, The Ohio State University Wexner Medical Center, Columbus, Ohio, USA

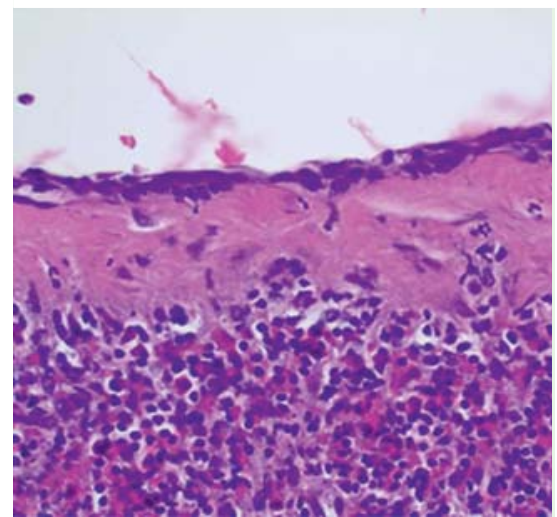

Fig. 2 Histopathology of the epidermoid cyst within an intrapancreatic accessory spleen (IPAS) showing a thin epithelial layer with ectopic splenic tissue.

\section{Acknowledgments $\nabla$}

This study was funded by the American College of Gastroenterology pilot research grant (S.K.; ClinicalTrials.gov: NCT02516488). 


\section{References}

1 Uchiyama S, Chijiiwa K, Hiyoshi M et al. Intrapancreatic accessory spleen mimicking endocrine tumor of the pancreas: case report and review of the literature. J Gastrointest Surg 2008; 12 : $1471-1473$

2 Krishna SG, Lee JH. Appraisal of needlebased confocal laser endomicroscopy in the diagnosis of pancreatic cysts. World J Gastroenterol 2016; 22: 1701-1710
Bibliography

Dol http://dx.doi.org/

10.1055/s-0042-117506

Endoscopy 2016; 48: E332-E333

(c) Georg Thieme Verlag KG

Stuttgart · New York

ISSN 0013-726X

\section{Corresponding author}

Somashekar G. Krishna, MD, MPH

Sections of Pancreatic Disorders

and Advanced Endoscopy

Division of Gastroenterology,

Hepatology and Nutrition

395 W. 12th Avenue, 2nd floor

Columbus

Ohio

USA

somashekar.krishna@osumc.edu 